\title{
Acute Pancreatitis in an Alcohol Prohibited Society
}

M. Anwar Hanid, M.R.C.P. (UK), MD (Wales), Mohamed A. Al-Karawi, F.A. (W. Germany), Abdulrahman E. Mohamed, M.R.C.P., DIP. VEN., D.T.M. \& H.,

Riyadh, Saudi Arabia

DOI: http://dx.doi.org/10.5915/21-2-13311

Abstrac1

In a retrospective study of patients admitted to the Riyadh Armed Forces Hospital over a 40 month period (September, 1982-December, 1985), there were 23 patients $10.03 \%$ of total admissions) admitted with acute pancreatitis. Pancreatitis was associated with gallstones (8), hyperlipidemia (3), mumps (I), post-operative pancreatitis following cholecystectomy (1), and hydatid cyst obstructing the common bile duct (1). There was only one case of well documented chronic alcohol consumption and no obvious cause could be found in the remaining eight patients. Eleven patients developed complications: jaundice (7), acute renal failure (3), and pulmonary complications (2). Two of these patients died. Two had recurrent pancreatitis and developed diabetes mellitus. There were very few patients with pancreatitis in this series. This is believed to be the result of virtually no alcohol consumption in our community.

Keywords: Pancreatitis, etiology, alcohol prohibition

The exact cause of acute pancreatitis is uncertain in some patients. However, there are certain well documented predisposing causes such as alcoholism,' hyperlipidemia, ${ }^{2}$ hypercalcemia, ${ }^{3}$ biliary tract disease, ${ }^{4,5}$ trauma, ${ }^{6}$ and familial inheritance. ${ }^{7}$ Within the Kingdom of Saudi Arabia, alcohol consumption is prohibited. We report a retrospective study of patients with acute pancreatitis who were admitted to the Riyadh Armed Forces Hospital over a 40 month period to show the relatively low incidence of alcoholism as an etiologic factor.

\section{Material and methods.}

Reviewed were 73,044 patients admitted to Riyadh Armed Forces Hospital over a 40 month period, September, 1982, through December, 1985. The following data were collected from the charts of those admitted with acute pancreatitis: age, sex, presenting symptoms, serum amylase levels, results

Froin the Department of Medicine, Armed Forces Hospiral, Riyadh, Saudi Arabia.

Reprint requests: A. E. Mohamed,

Armed Forces Hospital,

P.O. Box 7897,

Riyadh, 11159, Saudi Arabia. of investigations, e.g., sonography, cholecystogram, lipid profile, endoscopic retrograde cholangiopancreatography (ERCP) outcome, surgical treatment, and complications. An attempt to define the etiology was made.

\section{Results}

Twenty-three cases of acute pancreatitis $(0.03 \%)$ were admitted over this 40 month period. These were composed of 16 females (age range $12-79$ years, mean 51.8 years) and 7 males (age range $7-59$ years, mean 42.3 years). In all cases, the presentation was acute with abdominal pain, nausea, and vomiting. In all instances the serum amylase was over $600 \mathrm{IU} / \mathrm{L}$ (normal range $=0-100 \mathrm{IU} / \mathrm{L}$ ). In 21 out of 23 cases, the level was greater than $1,000 \mathrm{IU} / \mathrm{L}$. The diagnostic tests and etiology are shown in Tables 1 and 2, respectively.

The presence of gallstones was confirmed in nine patients, in one of which the pancreatitis occurred two days post-cholecystechtomy, and cholelithiasis was believed to be the causative factor. In three patients, hyperlipidemia was considered to be the predisposing factor. These three patients were known to have hyperlipidemia before their presentation with acute pancreatitis. A seven year old child presented with acute pancreatitis due to a mumps infection and one patient had a hydatid cyst obstructing the lower 
Table 1. Diagnostic tests

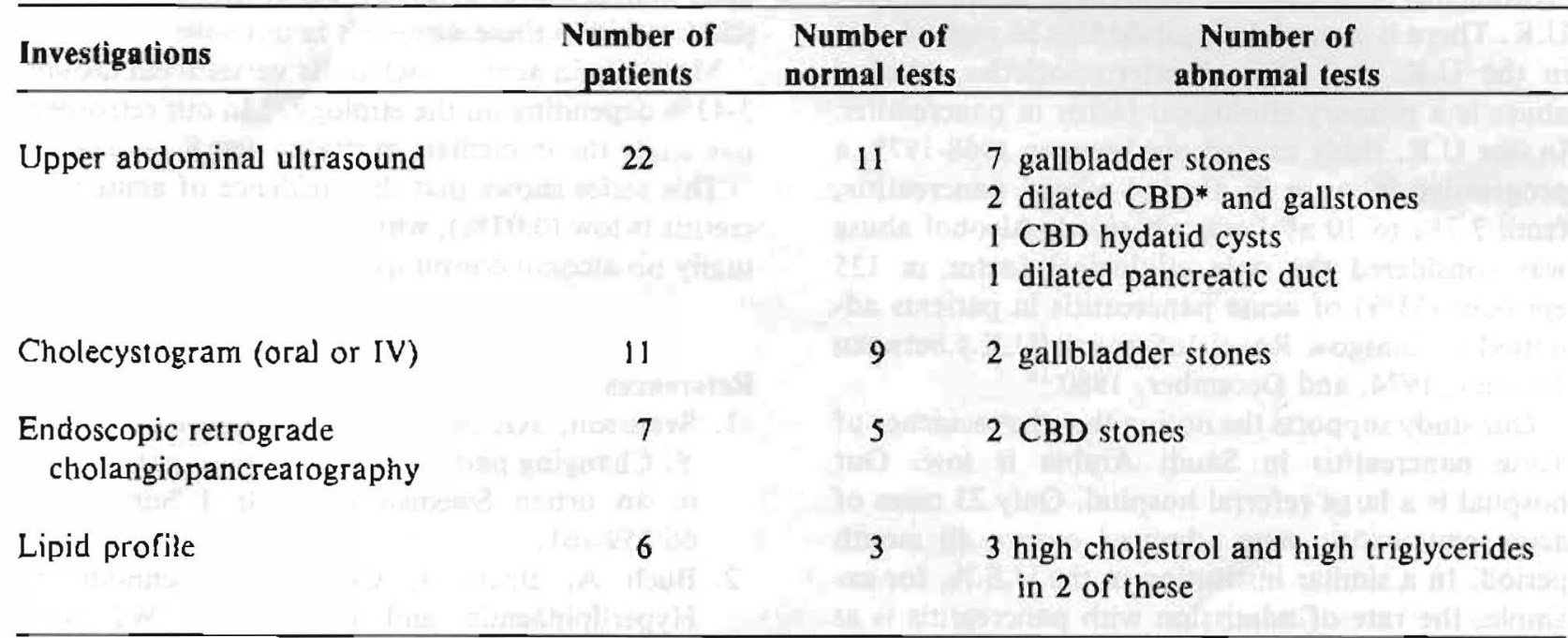

*Common bile duct.

Table 2. Etiology of acute pancreatitis in the study patients

\begin{tabular}{lcr}
\hline Etiology & $\begin{array}{c}\text { Number of } \\
\text { patients } \\
\text { (percent) }\end{array}$ & $\begin{array}{c}\text { Serum } \\
\text { amlyase* } \\
\text { (range) }\end{array}$ \\
\hline $\begin{array}{l}\text { Cholelithiasis } \\
\text { Post cholecystectomy }\end{array}$ & $8(35)$ & $600-5750$ \\
$\quad$ for gall stones & $1(4)$ & 2460 \\
Hyperlipidemia & $3(13)$ & $860-1680$ \\
Mumps & $1(4)$ & 1290 \\
CBD thydatid disease & $1(4)$ & 2538 \\
Alcohol & $1(4)$ & 21380 \\
Idiopathic & $8(35)$ & $1680-8429$ \\
\hline
\end{tabular}

*Normal range $0-100 \mathrm{IU} / \mathrm{L}$

tCommon bile duct.

end of the common bile duct. Only one of the patients had a history of chronic alcohol consumption. In the remaining eight cases, no obvious cause could be found.

Ultrasound examination of the upper abdomen was performed in all patients except for the child with mumps pancreatitis. A cholecytogram (oral or IV) was performed in 11 patients and gallbladder stones were identified in two. Endoscopic retrograde cholangiopancreatography (ERCP) was done in seven patients, two of whom had biliary pancreatitis as they had stones in the common bile duct which were extracted after papillotomy. The lipid profile was obtained in six patients and was abnormal in three.

Complications as a direct result of pancreatitis were documented in 11 patients. Seven patients were jaundiced at the time of presentation. Acute renal failure occurred in three patients, one of whom was also jaundiced. Basal collapse/consolidation was noted in two patients at the time of their admission.

Of the 23 patients, two died during hospitalization. One patient who underwent cholecystectomy for cholelithiasis was found to have acute hemorrhagic pancreatitis. Post-operatively the patient developed acute renal failure and a pancreatitic bed abscess and died. The second patient developed pancreatitis two days post-cholecystectomy for gallstones and subsequently developed multiple complications and died. A third patient died suddenly two weeks after being discharged, after having completely recovered from acute pancreatitis. Four patients were not available for follow-up after discharge. Sixteen patients were followed for a period that ranged from two to 30 months. Two patients were re-admitted with a second episode of acute pancreatitis and were well thereafter. One patient developed diabetes mellitus. The remaining 14 patients have been well and asymptomatic on follow-up.

\section{Discussion}

Acute pancreatitis is an acute inflammation of the pancreas, usually non-bacterial. While there are certain well-documented causes, the precise pathogenesis remains uncertain in many cases. Alcohol,' hyperlipidemia, ${ }^{2}$ hypercalcemia', cholelithiasis, ${ }^{4}$ post-gastric or biliary surgery, ${ }^{5}$ and trauma ${ }^{6}$ are some of the well known etiological factors. A rare familial type of pancreatitis has also been described.?

Alcohol may produce increased pancreatic secretion. In the face of pancreatic duct obstruction for 
proteinacious material, this can lead to gland autodigestion. The emphasis on alcohol-related pancreatitis has been reported from the U.S.A. ${ }^{8}$ and the U.K. There is accumulating evidence to suggest that in the U.K. and other Western societies, alcohol abuse is a primary etiological factor in pancreatitis. In one U.K. study carried out between 1968-1979, a progressive increase in alcohol-related pancreatitis, from $3.7 \%$ to $10.8 \% \%^{\circ}$ was observed. Alcohol abuse was considered the only etiological factor in 135 episodes (33\%) of acute pancreatitis in patients admitted to Glasgow Royal Infirmary (U.K.) between January, 1974, and December, 1980.10

Our study supports the notion that the incidence of acute pancreatitis in Saudi Arabia is low. Our hospital is a large referral hospital. Only 23 cases of acute pancreatitis were admitted over a 40 month period. In a similar institution in the U.S.A. for example, the rate of admission with pancreatitis is at least five times more common. Furthermore, the contribution of alcoholism to acute pancreatitis in our experience has been relatively minor. Pancreatitis in a setting of chronic alcohol abuse was seen in only one out of 23 patients. Although alcohol consumption can not be ruled out completely in the eight "idiopathic" cases, there was no historical, clinical, or other evidence to suggest that such was the case. This has to be contrasted with the U.S.A. experience where as many as $90 \%$ cases of pancreatitis are associated with alcohol consumption.

Gallstones were demonstrated in nearly one third of our patients. This is similar to the findings in the U.K. " but nearly half of that reported by Ranson in the U.S.A. ${ }^{3}$ A small stone causing obstruction at the ampulla results in reflux of bile into the pancreatic duct and initiates an acute inflammatory process, is one hypotheses. Biliary infection is also thought to be another precipitant factor. Whether a small stone causes transient obstruction initiating pancreatitis and then passes through the ampulla is questionable. This mechanism may explain some of the "idiopathic" cases.

Nine patients had cholelithiasis on ultrasonography. Two patients had an ERCP and papillotomy performed to decompress the biliary tree. Six patients underwent cholecystectomy, two of whom died.

The role of emergency biliary surgery in gallstone pancreatitis is debatable. ${ }^{12,14}$ More recently, it has been suggested that ERCP plus papillotomy may be enough to decompress the biliary system, enabling the patient to recover from the episode of acute pancreatitis rather than to subject them to surgery. ${ }^{15,16}$

In one patient, a hydatid cyst caused an obstruction at the ampulla with resultant pancreatitis. Once this had been removed surgically, the patient recovered. More recently, endoscopic removal of a hydatid cyst from the common bile duct (CBD) has been described. ${ }^{17}$

Pancreatitis has been associated with type I, IV, and $\mathrm{V}$ hyperlipoproteinemia. The pathogenesis of pancreatitis in these disorders is unknown.

Mortality in acute pancreatitis varies from around $3-43 \%$ depending on the etiology. ${ }^{18}$ In our retrospective study the immediate mortality was $8.7 \%(2 / 23)$.

This series shows that the incidence of acute pancretitis is low $(0.03 \%)$, which may well be due to virtually no alcohol consumption in our community.

\section{References}

1. Svensson, JO, Norback, B, Bokey EL, Edlund Y. Changing pattern of aetiology or pancreatitis in an urban Swedish area. Br J Surg 1979; 66:159-161.

2. Buch A, Buch, J, Carlsen A, Schmidt A. Hyperlipidaemia and pancreatitis. WJ Surg 1980; 4:307-314.

3. Ludwig, GD, Chaykin LD. Pancreatitis associated with primary hyperparathyroidism. Med Clin N Amer 1966; 50:1403-1418.

4. Ranson JHC. Etiology and management of acute pancreatitis. In Surgical Diseases of the Pancreas; Ed. Brooks J, Philadelphia: W.B. Saunders, 1983, pp 146-181.

5. Banks, P.A. Pancreatitis. New York: Plenum Medical Book Company, 1979.

6. Northrup WF, Simmons RL. Pancreatic trauma: A review. Surgery 1972; 71:27-43.

7. Sato T, Saitoh Y. Familial chronic pancreatitis associated with pancreatic lithiasis. Am H Surg 1974; 127:511-517.

8. Durr GH. Acute pancreatitis. In Exocine Pancreas. Eds. Howat, H F, and Sarles, H. London: W.B. Saunders; pp 352-401.

9. Corfield AP, Cooper MJ, Williamson RC. Acute pancreatitis: A lethal disease of increasing incidence. Gut 1985; 26:724-729.

10. Blamy SL, Imrie CW, O'Neill J, Gilmour WH, Carter DC. Prognostic Factors in Acute Pancreatitis.

11. Trapnell JE. Acute Pancreatitis in Great Britain -a review. Med J Aust 1974; 2:450-455.

12. Ranson JHC. The timing of biliary surgery in acute pancreatitis Ann Surg 1979; 189:654-663.

13. Acosta JM, Pellegrini CA, Skinner DB. Etiology and pathogenesis of acute biliary pancreatitis. Surgery $1980 ; 88: 118-125$.

14. Stone HH, Fabian TC, Dunlop WE. Gallstone pancreatitis: Biliary tract pathology in relation to time of operation. Ann Surg 1981; 194:305-313.

15. Safrany L, Cotton PB. A preliminary report: Urgent duodenoscopic sphincterotomy for acute gallstone pancreatitis. Surgery 1981; 89:424-428.

16. Van Der Spuy S. Endoscopic sphincterotomy in the management of gallstone pancreatitis. En- 
doscopy $1981 ; 13: 25-26$

17. Al Karawi M, Hanid MA. Endoscopic removal of daughter echinococcus cysts from the common bile duct. Hepato-Gastroenterol 1985; 32:296-298.
18. Ranson JHC. Etiology and prognostic factors in human acute pancreatitis: a review. Am J Gastroenterol 1982; 77:633-638.

19. Al Bar, M. JIMA (in press). 